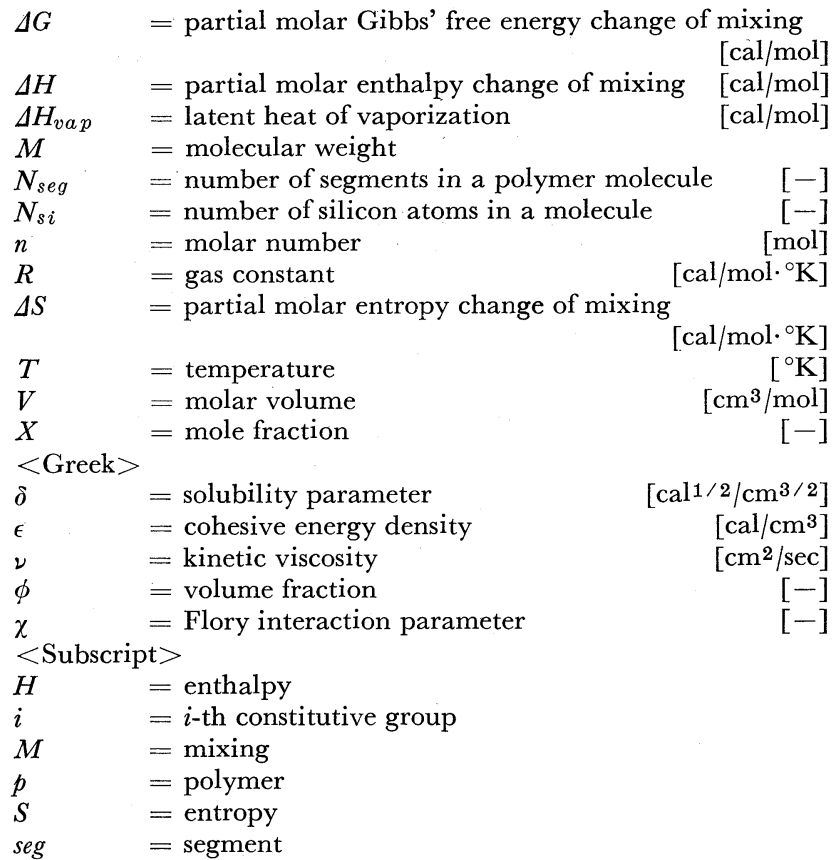

\section{Literature Gited}

1) Blanks, R. F. and J. M. Prausnitz: Ind. Eng. Chem., Fundamentals, 3, 1 (1964)

2) Burrell, H.: Official Digest, 27, 726 (1955)

3) Flory, P. J.: "Principles of Polymer Chemistry", Cornell Univ. Press, N. Y. (1953)

4) Fujii, M. (Ed.): "High Polymer Engineering, vol. XIII, Properties and Uses of Polymer(I)", Chijin-shokan, Tokyo (1969)

5) Glasstone, S., Laidler, K. J. and H. Eyring: "The Theory of Rate Processes", McGraw-Hill, N. Y. (1941)

6) Hildebrand, J. H. and R. L. Scott: "Solubility of Non-electrolytes", Reinhold Publ. Corp. (1950); Hildebrand, J. H., J. M. Prausnitz and R. L. Scott: "Regular and Related Solutions", van Nostrand Reinhold (1970)

7) Lieberman, E. P.: Official Digest, 34, 30 (1962)

8) Rochow E. G.: "An Introduction to the Chemistry of the Silicones", John Wiley \& Sons Inc. N. Y. (1951)

9) Scott, R. and M. Magat: J. Polymer Sci., 4, 553 (1949)

10) Shin-etsu Silicone Manual P6-3A; Shin-Etsu Silicon Technical Data T6-4

11) Shinoda, K.: "Solution and Solubility", Maruzen, Tokyo (1966)

12) Small, P. A : J. Appl. Chem., 3, 71 (1953)

13) Watanabe, H. and T. Miyauchi: to be submitted

14) Wilcock, D. F.: J. Am. Chem. Soc., 68, 691 (1946)

\title{
DIFFUSION OF NON-POLYMERIC AND POLYMERIC MOLECULE IN CHAIN POLYMER LIQUID
}

\author{
A THEORETICAL EXPLANATON OF ANOMALOUS \\ BEHAVIOR OF DIFFUSIVITY IN POLYMERIC SYSTEM*
}

\author{
Hisahiko WATANABE and Terukatsu MiYAUChI \\ Department of Chemical Engineering, University of Tokyo, \\ Tokyo, Japan
}

\begin{abstract}
This work is concerned with the diffusivity in polymeric two-component systems in which the penetrant concentration is infinitely dilute. In polymeric systems the WilkeChang equation does not hold, and diffusivity shows an anomalous behavior; that is, it shows a constant value regardless of medium molecular weight. This anomaly is accounted for in this work.

The diffusivity of non-polymeric and polymeric molecules through a polymeric medium has been derived theoretically as an extension of the free-volume theory by Bueche. With this formulation, a simple expression has been developed to correlate diffusivity, viscosity, temperature and molecular weight of the system. Good agreement with the experimental data for the polydimethylsiloxane system is obtained. It is found that $D_{\eta} / T$ is proportional to $M^{3.5}$ for the range of $M>M_{b}$, and to $M$ for the range of $M<M_{b}$, where $M$ is the molecular weight of polymeric medium and $M_{b}$ is a characteristic value.
\end{abstract}

* Received on July 28, 1972

T113 東京都文京区本郷7-3-1

東京大学工学部化学工学科 渡辺尚彦

\section{Introduction}

There have been a great number of theoretical as 


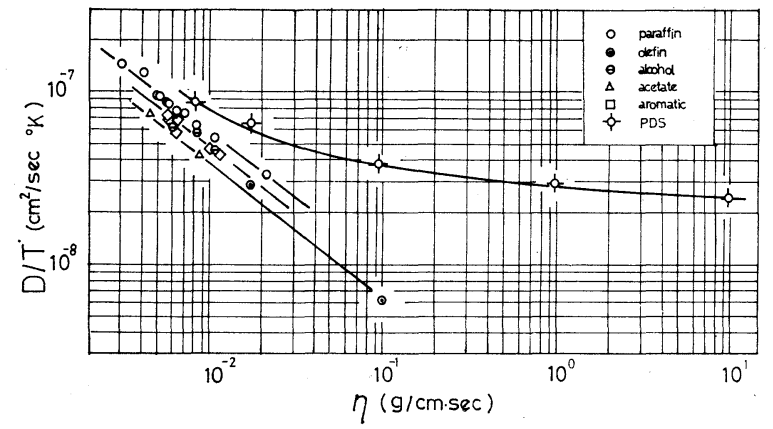

Fig. 1 The diffusivity of iodine in various solvents vs. solvent viscosity ${ }^{35}$ )

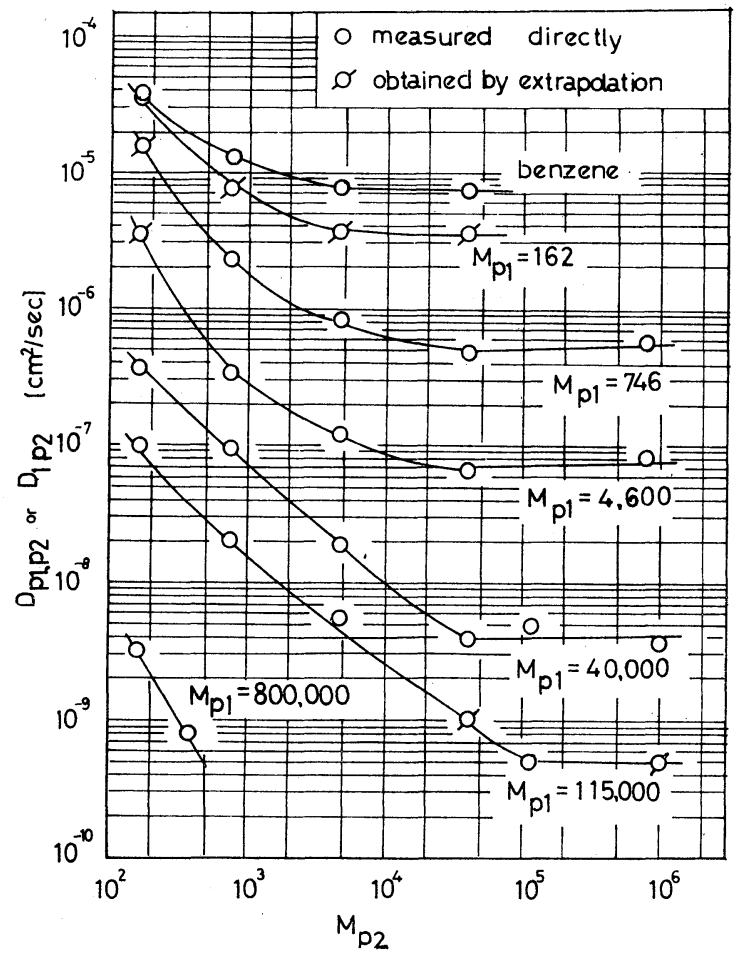

Fig. 2 The diffusivity of benzene and PDS in PDS matrix. $\left.{ }^{33}\right) M_{p 2}$ is the molecular weight of medium PDS.

well as experimental investigations of diffusivity in non-polymeric liquid phase. For one-component selfdiffusivity, the theory of absolute processes by Eyring and his co-workers ${ }^{10,13)}$ its modifications ${ }^{20,22,26)}$ and others ${ }^{4,8,17,27)}$ have been presented. Among these, Eyring's equation is the simplest in form:

$$
\frac{D_{\eta}}{T}=\frac{k}{6}\left(\frac{N_{A}}{V}\right)^{1 / 3}
$$

For two-component systems, however, theoretical treatments ${ }^{12,15,18,23)}$ have some difficulties in practical use. Therefore, empirical correlations, such as Wilke and Chang's equation ${ }^{36,37)}$, its modifications ${ }^{11,25,29,32)}$ and others ${ }^{16,19,21,24,31)}$ are commonly used. The WilkeChang equation is as follows:

$$
\frac{D_{12} \eta_{2}}{T}=7.4 \times 10^{-10} \frac{\left(b M_{2}\right)^{1 / 2}}{V_{1}^{0.6}}
$$

\footnotetext{
* PDS is a linear chain polymer consisting of siloxane units. $\left(\mathrm{CH}_{3}\right)_{3} \mathrm{Si}-\left(-\mathrm{OSi}\left(\mathrm{CH}_{3}\right)_{3}-\right)_{n-1} \mathrm{CH}_{3}$
}

When the size of penetrant molecule is sufficiently large compared with that of medium liquid, the Stokes-Einstein equation ${ }^{9)}$ can be applied:

$$
\frac{D_{12} \eta_{2}}{T}=\frac{k}{6 \pi r_{1}}
$$

By the use of the results of these investigations above, diffusivities of one- and two-component non-polymeric systems are predictable within about 50 per cent error.

On the contrary, anomalous behavior of diffusivity has been reported for viscous liquid ${ }^{19,21)}$, in particular for highly polymeric medium ${ }^{33,35)}$. Most of all, the experimental systems used for Wilke-Chang's correlation are less viscous than a few centipoise ${ }^{36,37)}$. For more viscous systems, Wilke-Chang's equation gives fairly smaller values than those of experiment $t^{14,19)}$.

This tendency turns out to be decisive when the diffusion medium is composed of chain polymer molecules ${ }^{33,35)}$. The diffusivities $D_{12}$ of iodine (1) in various kinds of liquid medium (2), available in the literature, are plotted against the medium viscosity $\eta_{2}$ in Fig. $1^{35)} \cdot \ln \left(D_{12} / T\right)$ vs. $\ln \eta_{2}$ plot for each nonpolymeric homologue liquid shows parallel straight line, with a gradient of -0.7 . (This implies the relation $\eta \propto M^{1.2}$ in Wilke-Chang's correlation.) However, the diffusivity of iodine in polydimethylsiloxane* (PDS) behaves in an entirely different manner, approaching a constant regardless of medium molecular weight, when the polymer chain length grows sufficiently high $^{35)}$. The diffusivity of benzene in PDS is also reported ${ }^{33)}$ to show similar behavior (Fig. 2). Tanner ${ }^{33)}$ measured the diffusivity of PDS in PDS, varying molecular weight of penetrant and medium component and also obtained a constant diffusivity region (see Fig. 2). These have common characteristics: (i) When the medium molecular weight $M_{p 2}$ does not reach a critical value, diffusivity $D_{12}$ depends on medium molecular weight $M_{p 2}$, but is considerably insensitive compared with that in non-polymeric system. (ii) When $M_{p 2}$ exceeds a certain critical molecular weight, the diffusivity remains constant regardless of molecular weight $M_{p 2}$.

This anomalous behavior of diffusivity in polymeric systems cannot be interpreted by the use of theoretical nor empirical equations for non-polymeric systems. This originates in the constitutional difference between polymeric and non-polymeric systems.

A number of investigations have been presented ${ }^{1,6)}$ on physical properties of polymer. However, most quantitative treatments of diffusivity contain some physical quantities which are not available in common. As an exception, a simple relationship correlating diffusivity, viscosity, temperature and molar volume was given by Bueche ${ }^{1}$ ) for one-component self-diffusivity (see section 2.3). This work extends that of Bueche to two-component systems in which penetrant molecule is very dilute. In the next section, the treatment of chain polymer by a hole model is described. Then, in section 3, two-component diffusivity equations in polymeric systems are derived by the use of 
free-volume theory. A fairly complete explanation of the anomalous behavior as pointed out above is given in section 4 , using the equations derived in section 3.

\section{Description of the Behavior of Chain Poly- mer by Hole Model}

\section{1 Diffusivity of small molecule and chain polymer molecule}

Let us consider a simple liquid composed of small molecules. The diffusivity of a molecule is given as follows ${ }^{1)}$ :

$$
D=\phi \delta^{2} / 6
$$

where $\phi$ is the mean jump frequency and $\delta$ is the mean jump distance of the molecule. Eq.(4) holds good not only for one-component system but also for two-component system, as long as the system is infinitely dilute. This is because when the system is sufficiently dilute, the diffusion medium can be regarded as homogeneous. Consequently, a mean jump distance $\delta$ and a mean jump frequency $\phi$ of penetrant molecule can be uniquely defined.

The method used to derive diffusivity in non-polymeric liquid described above can be extended to chain polymer systems with ease ${ }^{1)}$. Let us suppose that a chain polymer molecule consists of $N$ segmental units; each segment is $a$ in length and is composed of several repeating molecular units. When we suppose, further, that these segments are completely freely oriented and make jumps independently in each other, the self-diffusivity $D_{p p}$ of one-component polymer liquid is obtained $^{1)}$ as follows, substituting the jump frequency $N \phi_{s}$ and jump distance $\delta_{s} / N$ of a polymer molecule to Eq.(4):

$$
D_{p p}=\phi_{s} \delta_{s}^{2} / 6 N
$$

where $\phi_{s}$ and $\delta_{s}$ are jump frequency and jump distance of segmental units, respectively.

\section{2 The effect of entanglement}

In the previous section, the motion of each polymer molecule is assumed independent of each other without any entanglement. However, we must consider the effect of entanglement when molecular weight of polymer grows sufficiently high. In general, Eq.(5) is also applicable to entangled chain polymer systems ${ }^{11}$, provided $N$ is replaced by a modified value $N^{*}$, which is larger than $N$.

$$
D_{p, p}=\phi_{s} \delta_{s}^{2} / 6 N^{*}
$$

According to Bueche ${ }^{1,3)} N^{*}$ is approximated as follows

(a) $M_{p}<2 M_{e}$ :

(b) $M_{p}>2 M_{e}$ :

$$
N^{*} f_{o s}=N f_{o s}
$$

$$
\begin{aligned}
N^{*} f_{o s}= & N f_{o s}\left(\rho N_{A} / 48\right)\left(M / M_{e}\right)^{2} M^{1 / 2}\left(a^{2} / M_{s e g}\right)^{3 / 2} \\
& \times \sum_{n=1}^{\infty} \sigma^{n}(2 n-1)^{3 / 2}
\end{aligned}
$$

where $f_{o s}$ is the friction factor of a segmental unit, and is related to diffusivity by

$$
D_{p, p}=k T / N^{*} f_{o s}
$$

$\sigma$ is slippage factor. $M$ and $M_{\text {seg }}$ are, respectively, the molecular weight of polymer molecule and that of segmental unit. $M_{e}$ is a kind of critical molecular weight, corresponding to several segmental units between entangling points. The comparison of Eqs.(6) and (8) gives

$$
f_{o s}=6 k T / \phi_{s} \delta_{s}^{2}
$$

2. 3 A simple relation between diffusivity and viscosity (one-component polymeric system)

The viscosity of polymer solution and polymer melt is given as follows ${ }^{1,7)}$ :

$$
\eta_{p}=\left(\rho N_{A} / 36\right)\left(a^{2} / M_{s e g}\right) N^{*} f_{o s}
$$

When we combine the equation of diffusivity Eq.(8) and that of viscosity Eq.(10) for polymeric system, a simple equation is obtained as follows ${ }^{1)}$ :

$$
\frac{D_{p, p} \eta_{p}}{T}=\left(\frac{k \rho N_{A}}{36}\right)\left(\frac{a^{2}}{M_{s e g}}\right)=\frac{k}{36}\left(\frac{N_{A}}{V_{s e g}}\right)^{1 / 3}
$$

Eq.(11) is similar to Eq.(1) for non-polymeric liquid. It should be noticed, however, that the right-hand side is not a function of molar volume of the whole polymer molecule but that of a segmental unit. Eq. (11) says that $D_{p, p} \eta_{p} / T$ will be constant for a homologue polymeric series.

The relation $D_{p, p} \eta_{p} / T=$ constant, is experimentally observed $^{11}$ in both condensed polymeric solution and polymer melt of poly- $n$-butyl acrylate, polystyrene and polymethylene.

\section{Two-component Diffusivity in Chain Poly- mer Liquids}

\section{1 The expression of mean jump frequency by free-volume theory}

The simple equation relating the diffusivity and viscosity of polymeric system described in the previous section is confirmed to one-component system. The objective of this work is to extend this kind of equation to two-component systems. Diffusivity is generally given by Eq.(4). It is unlikely that the distance $\delta$ which a molecule travels in jumping from one equilibrium position to another can vary too greatly from material to material. Therefore the variation of diffusivity from system to system, often many orders of magnitude, is mainly the result of the different value of jump frequencies ${ }^{1)} \phi$.

According to the free volume theory of Bueche ${ }^{1,2)}$ and Cohen and Turnbull ${ }^{5)}$, jump frequency $\phi$ of a small molecule or a segment is considered to be proportional to the probability that the molecule or the segment is adjacent to a hole which is larger than a certain critical volume $v^{*}$. Since this probability is proportional to $\exp \left(-\beta^{*} v^{*} / v_{f}\right)$ over a glass-transition temperature, jump frequency $\phi$ is given in the end as follows by the free-volume theory ${ }^{1)}$ :

where

$$
\phi=\phi_{o} \exp \left(-\beta^{*} v^{*} / v_{f}\right)
$$

$$
\beta^{*}=\ln \left(v^{*} / v_{f}\right)-1
$$

$v_{f}$ is a mean free volume per a molecule or a segment $\left(V_{f} / N_{A}\right)$ and $\beta^{*}$ is considered to be a constant value near $1^{1)}$. 


\section{2 Diffusivity in polymeric medium-Gase I: diffusivity of a small molecule-}

This section concerns the diffusivity of a small molecule, the size of which is about equal to that of a segmental unit of the medium polymer molecules. The expression of jump frequency $\phi$ given above is that for one-component system. For the treament of two-component diffusion processes, however, the individual role of penetrant and medium molecule should be appropriately distinguished. In Eq.(12), the critical free volume $v^{*}$ must be a quantity concerned with penetrant molecule (1), while the mean free volume $v_{f}$ is concerned with medium molecule $(p 2)$. The probability constant $\phi_{0}$ may be interpreted as a limit of $\phi$ when $v_{f}$ approaches infinity. Therefore, $\phi_{0}$ may be concerned with penetrant molecule (1). Based on these considerations, the diffusivity $D_{1, p 2}$ and jump frequency $\phi_{1, p 2}$ of molecule (1) in chain polymer medium ( $p 2)$ may be expressed as follows:

$$
\begin{gathered}
D_{1, p 2}=\phi_{1, p 2} \delta_{s}^{2} / 6 \\
\phi_{1, p 2}=\phi_{o 1} \exp \left(-\beta^{*} v_{1}^{*} / v_{f s}\right)
\end{gathered}
$$

On the other hand, the jump frequency of segmental unit of polymer is given by Eq.(12):

$$
\phi_{s}=\phi_{o s} \exp \left(-\beta^{*} v_{s}^{*} / v_{f s}\right)
$$

Now, let us take the ratio of diffusivities $D_{1, p 2}$ and $D_{p 2, p 2}$ to cancel unknown parameters, rearranging it by the use of Eqs.(15) and (16):

$$
\begin{aligned}
\frac{D_{1, p 2}}{D_{p 2, p 2}} & =\frac{\phi_{1, p_{2}} \delta_{s}^{2} / 6}{\phi_{s} \delta_{s} / 6 N^{*}}=\frac{\phi_{1, p 2}}{\phi_{s}} N^{*} \\
& =\frac{\phi_{o 1}}{\phi_{o s}} N^{*} \exp \left\{-\beta^{*}\left(v_{1}^{*}-v_{s}^{*}\right) / v_{f s}\right\}
\end{aligned}
$$

When the size of penetrant molecule is approximately equal to that of a segmental unit of the polymer medium, we can substitute $v_{1}^{*} \approx v_{s}^{*}$ and further $\phi_{01} \approx$ $\phi_{o s}$ to Eq. (17) to obtain

$$
\frac{D_{1, p 2}}{D_{p 2, p 2}} \approx N^{*}
$$

Eliminating $D_{p 2, p 2}$ by the use of Eq.(11), we can obtain a simple equation relating diffusivity $D_{1, p_{2}}$ and medium viscosity $\eta_{p_{2}}$ :

$$
\frac{D_{1, p 2} \eta_{p 2}}{T} \approx \frac{k N^{*}}{36}\left(\frac{N_{A}}{V_{\text {seg }}}\right)^{1 / 3}
$$

Consequently, the diffusivity $D_{1, p 2}$ of a small molecule (1), the size of which is nearly equal to that of a segmental unit of medium, through homologue series of chain polymer liquid medium ( $p 2)$, is given as follows, according to the classification for entanglement effect given by Eq.(7) :

(a) When $M_{p 2}<2 M_{e}: N^{*}$ is given by $N^{*}=N=$ $M_{p} / M_{\text {seg }}$;

The result of substitution to Eq.(19) reduces to

$$
\frac{D_{1, p 2} \eta_{p 2}}{T} \propto M_{p 2}
$$

(b) When $M_{p 2}>2 M_{e} ; N^{*}$ is proportional to $M_{p}^{3.5}$, substituting $N=M_{p} / M_{\text {seg }}$ in Eq.(7b).

The result of substitution to Eq.(19) reduces to

$$
\frac{D_{1, p 2} \eta_{p 2}}{T} \propto M_{p 2}^{3.5}
$$

\section{3 Diffusivity in polymeric medium-Gase II:} diffusivity of a chain polymer molecule -

In this section, we deal with the case where the diffusing molecule is not a small molecule but a homologue polymeric one, consisting of $N_{p 1}$ segmental units, while the polymeric medium has $N_{p 2}$ segmental units in a molecule. Let us repeat that Eq.(5) is derived by considering the jump frequency and jump distance of the center of mass of penetrant polymer molecule. Therefore, $N$ in Eq.(5) and $N^{*}$ in Eq.(6) are quantities concerned with penetrant polymer molecule. Consequently, the diffusivity $D_{p 1, p 2}$ is given as follows:

$$
D_{p 1, p 2}=\phi_{s} \delta_{s}^{2} / 6 N_{p 1}^{*}
$$

Although Eq.(21) is good for non-entangling region, it may not be strictly correct to apply Eq.(7) as the entanglement effect $N_{p 1}^{*}$ to two-component diffusivity $D_{p 1, p 2}$ (Eq.(21)), since the molecular weight of penetrant is different from that of medium molecule. However, as a first approximation, let us use Eq.(7) as the entangling effect for two-component system, too. On the other hand, one-component self-diffusivity $D_{p 2}, p 2$ is given as follows:

$$
D_{p 2, p 2}=\phi_{s} \delta_{s}^{2} / 6 N_{p 2}^{*}
$$

The ratio of these two diffusivities reduces to

$$
\frac{D_{p 1, p 2}}{D_{p 2, p 2}}=\frac{N_{p 2}^{*}}{N_{p 1}^{*}}
$$

Eliminating $D_{p 2, p 2}$ by the use of Eq.(11), we obtain

$$
\frac{D_{p 1, p 2} \eta_{p 2}}{T}=\frac{k}{36}\left(\frac{N_{A}}{V_{\text {seg }}}\right)^{1 / 3} \frac{N_{p 2}^{*}}{N_{p 1}^{*}}
$$

where $N_{p 1}^{*}$ and $N_{p 2}^{*}$ are given by Eq.(7). According to the molecular weight classification for entanglement effect, Eq.(24) is rewritten as follows:

(a) $M_{p 1}<2 M_{e}$ and $M_{p 2}<2 M_{e}$ :

$$
\begin{aligned}
& \frac{D_{p 1, p 2} \eta_{p 2}}{T} \propto \frac{M_{p 2}}{M_{p 1}} \\
& \text { (b) } M_{p 1}<2 M_{e} \text { and } M_{p 2}>2 M_{e} \text { : } \\
& \frac{D_{p 1, p 2} \eta_{p 2}}{T} \propto \frac{M_{p 2}^{3.5}}{M_{p 1}} \\
& \text { (c) } M_{p 1}>2 M_{e} \text { and } M_{p 2}<2 M_{e} \text { : } \\
& \frac{D_{p 1, p 2} \eta_{p 2}}{T} \propto \frac{M_{p 2}}{M_{p 1}} \\
& \text { (d) } M_{p 1}>2 M_{e} \text { and } M_{p 2}>2 M_{e} \text { : } \\
& \frac{D_{p 1, p 2} \eta_{p 2}}{T} \propto \frac{M_{p 2}^{3.5}}{M_{p 1}^{3.5}}
\end{aligned}
$$

where in case (c), the entangling effect of penetrant molecule is neglected because of sufficiently dilute concentration. In case (d), entanglement effect is considered, since the dilute penetrant molecule $(p 1)$ may be sure to entangle with medium molecules $(p 2)$; however, if $M_{p 1} \gg M_{p 2}$, the effect of entanglement will be considerably decreased to give lower power order with $M_{p 1}$; and if $M_{p 1} \ll M_{p 2}$, to give higher power order.

\section{Discussion: Comparison with Experimental Data}

In this section, the theoretical equations derived in 


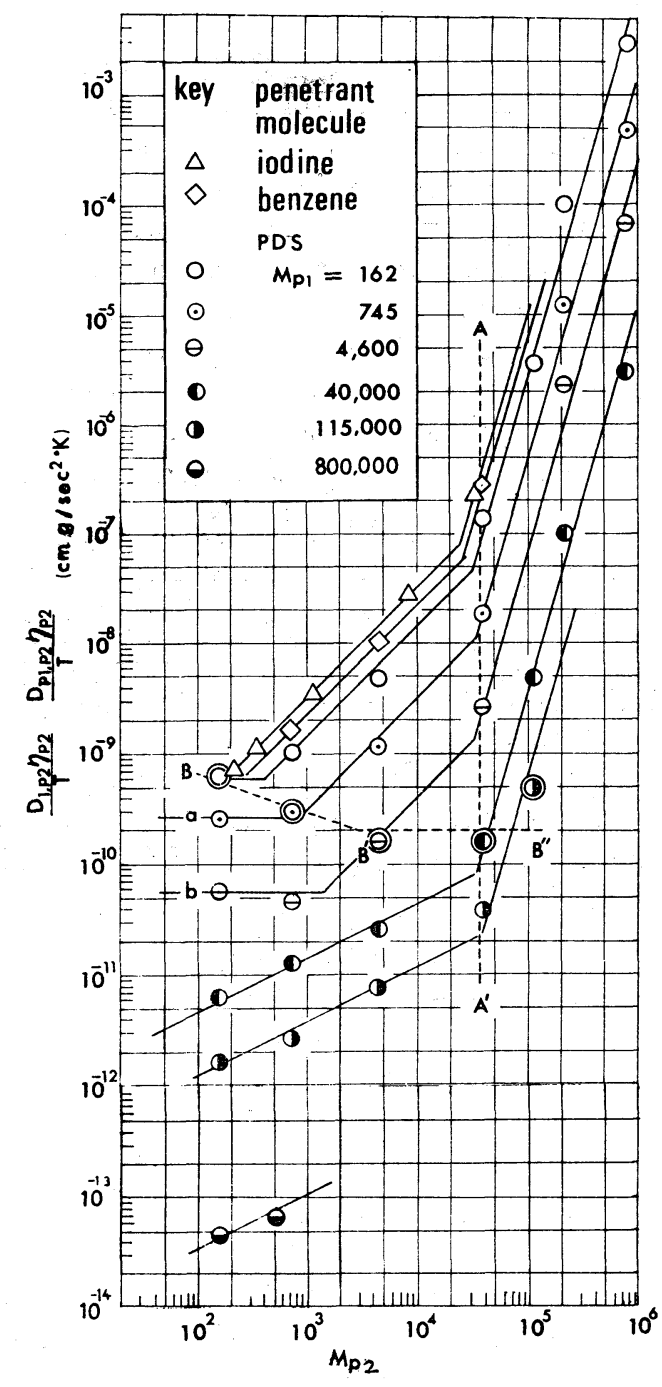

Fig. 3 Plot of diffusivity in PDS homologue liquids as $\ln D_{\eta} / T$ vs. $\ln M_{p 2}$.

$M_{p 2}$ is the molecular weight of medium PDS. Encircled points are one-component selfdiffusivities.

the previous section are compared with experimental data. And the anomalous behavior in polymeric systems as pointed out in section 1 is theoretically explained.

\section{1 A plot of literature data for comparison}

There are only a few data available in the literature for our discussion. The diffusivity data of iodine ${ }^{35)}$, benzene ${ }^{33)}$ and polydimethylsiloxane ${ }^{33)}$ (PDS) in medium PDS are plotted $\ln \left(D_{1, p_{2}} \eta_{p 2} / T\right)$ vs. $\ln M_{p 2}$ or $\ln \left(D_{p 1, p 2} \eta_{p 2} / T\right)$ vs. $\ln M_{p 2}$ in Fig. 3, to be compared with theoretically derived equations above. The constant temperature at which diffusivity was measured is $33^{\circ} \mathrm{C}$ for both benzene-PDS and PDS-PDS systems ${ }^{34)}$, and is $25^{\circ} \mathrm{C}$ for iodine-PDS system ${ }^{35)}$. The viscosity data of PDS are found in manuals ${ }^{30}$.

Each $D_{\eta} / T$ vs. $M_{p 2}$ curve is divided into three regions by two border lines; namely a straight line $\mathrm{AA}^{\prime}$ $\left(M_{p 2}=37,000\right)$, which is the critical value $2 M_{e}$ of PDS (see section 4.3), and a curve $\mathrm{BB}^{\prime} \mathrm{B}^{\prime \prime}$ which represents one-component self-diffusivity. The features of each region are summarized as follows:

In the right-hand region of the straight line $\mathrm{AA}^{\prime}$, $D_{\eta} / T$ is proportional to $M_{p 2}^{3.5}$. In the wedge-shaped region intercepted by the straight line $\mathrm{AA}^{\prime}$ and the curve $\mathrm{BB}^{\prime} \mathrm{B}^{\prime \prime}, D_{\eta} / T$ is nearly proportional to $M_{p 2}$. In the left-hand region of the curve $\mathrm{BB}^{\prime} \mathrm{B}^{\prime \prime}, D_{\eta} / T$ reduces to a constant regardless of $M_{p 2}$, provided $M_{p 1}$ is less than $2 M_{e}$. On the other hand, when $M_{p 1}$ exceeds the critical molecular weight $2 M_{e}$, the wedge-shaped region disappears and $D_{\eta} / T$ is no longer constant, but is proportional to $M_{p 2}^{1 / 2}$ in the left-hand region of the straight line $\mathrm{AA}^{\prime}$.

For the case of the diffusivity of iodine and benzene in PDS, Fig. 3 shows the proportionality of $D_{\eta} / T$ to $M_{2 p}$ in the wedge-shaped region and shows a transitional behavior to 3.5 power region at $M_{p_{2}}=40,000$.

\section{2 Comparison with the theory}

The three regions pointed out above completely agree with theoretical results given in the previous sections. The straight line $\mathrm{AA}^{\prime}$ corresponds to the critical molecular weight $2 M_{e}$ of entanglement effect. In the region where $M_{p 2}$ is larger than $2 M_{e}, D_{\eta} / T$ is proportional to $M_{p 2}^{3.5}$, as shown by Eqs. (20b), (25b) and $(25 \mathrm{~d})$. On the other hand, the curve $\mathrm{BB}^{\prime} \mathrm{B}^{\prime \prime}$ corresponds to the one-component self-diffusivity equation, Eq.(1) and Eq.(11). In the non-polymeric range, $D_{\eta} / T$ decreases in proportion to $V^{-1 / 3}$, namely to $M^{-1 / 3}$, since the density of PDS is nearly constant $(0.9$ $\left.1.0 \mathrm{~g} / \mathrm{cm}^{3}\right)$ regardless of degree of polymerization. In the polymeric range where medium polymer has enough molecular weight to reach a constant segment size, $D_{\eta} / T$ reduces to a constant regardless of $M_{p 2}$.

In the wedge-shaped region between the curve $\mathrm{BB}^{\prime} \mathrm{B}^{\prime \prime}$ and the straight line $\mathrm{AA}^{\prime}$, the proportionality between $D_{\eta} / T$ and $M_{p 2}$ corresponds to Eqs. (20a) and (25a). In the left-hand region of the curve $\mathrm{BB}^{\prime} \mathrm{B}^{\prime \prime}$, $D_{\eta} / T$ becomes constant regardless of $M_{p_{2}}$ (or $M_{2}$ ). This is the characteristic feature of the Stokes-Einstein equation (Eq.(3)).

On the contrary, when $M_{1}$ exceeds $2 M_{e}$, the wedgeshaped region disappears and the Stokes-Einstein equation is no longer satisfied for two cases: $M_{1}=$ 40,000 and $M_{1}=115,000$ in Fig. 3. This region corresponds to the case (c) in section 3.3, and Eq. (25c) should be satisfied, when the theory derived in this work holds good in this region. Eq.(25c) says that $D_{\eta} / T$ is proportional to $M_{p_{2}}$. Therefore, the behavior of experimental data, namely $D_{\eta} / T \propto M_{p 2}^{1 / 2}$, can be regarded as intermediate between the Stokes-Einstein equation and Eq. (25c). There is no detailed explanation for this discrepancy yet. However, some qualitative explanations such as what follows are possible:

The penetrant high polymer molecules may diffuse through small molecular medium, forming coils with its long chain segments. The entanglement effect of penetrant polymer may happen to fasten the coils to decrease its effective frictional force in diffusion process. Then the diffusivity may be increased, and $D_{\eta} / T$ might deviate from Eq.(25c). On the other hand, 
penetrant polymers cannot form coils when medium molecules are not small; therefore, Eq.(25d) holds good.

The concentration of penetrant molecules is 10 per cent weight fraction in benzene-PDS and PDS-PDS systems ${ }^{33}$. Although this does not seem to be dilute enough to satisfy the no-interaction assumption concerning penetrant molecules, good agreement between theory and experiment is obtained. The concentration of penetrant in iodine-PDS system is less than 1 per cent (weight) ${ }^{35}$.

\section{3 The explanation of constant diffusivity}

The remarkable feature of constant diffusivity in polymeric system corresponds to $D_{\eta} / T \propto M_{p 2}^{3.5}$ in our equation. The viscosity of condensed polymer solution and polymer melt is known ${ }^{1)}$ to be proportional to $M^{3.5}$ without exception in the range of higher molecular weight over a critical value $M_{b}$.

$$
\eta \propto M^{3.5} \quad\left(M>M_{b}\right)
$$

This critical molecular weight $M_{b}$ is approximately equal to $2 M_{e}{ }^{1)}$. Substituting Eq.(26) to Eqs.(20b), (25b) and (25d), the diffusivity $D_{1, p 2}$ or $D_{p 1, p 2}$ for the range of sufficiently large molecular weight medium reduces to:

$$
\begin{aligned}
D_{1, p 2}\left(\text { or } D_{p 1, p 2}\right) & =(\text { constant }) \frac{M_{p 2}^{3.5}}{\eta_{p 2}} \cdot T \\
& =(\text { constant })^{\prime} T
\end{aligned}
$$

Eq.(27) indicates a constant diffusivity at constant temperature regardless of medium molecular weight $M_{p 2}$. Therefore, the critical molecular weight for constant diffusivity may be equal to $2 M_{e}$ or $M_{b}$, which is 37,000 for PDS ${ }^{1)}$. However, when $M_{p 1}$ exceeds $2 M_{e}$ or $M_{b}$, the critical value for constant diffusivity seems to be $M_{p 1}$ itself as shown in Fig. 2.

\section{4 The ideal characteristic of PDS}

As is shown in the previous sections, the experimental result in PDS system considerably well satisfies the theoretical equations derived in this work. This good agreement may be attributed to the distinguished characteristics of PDS as a long-chain polymer molecule. That is, the segmental motion of PDS molecule is nearly completely freely oriented. This is supported by the fact that the activation energy for viscous flow of PDS is known to be peculiarly low compared with other substances, e.g. $n$-paraffins ${ }^{28}$. Furthermore, this free motion of segments of PDS was confirmed by measurement of dipole moment and electron diffraction $^{28)}$. Moreover, PDS is considered to have low glasstransition temperature $T_{g} ; T_{g}$ of silicon rubber is $-123^{\circ} \mathrm{C}^{1)}$. Therefore, room temperature is sufficiently high over $T_{g}$ for PDS system. Consequently, PDS system may be regarded as an ideal system for the theory based on completely freely oriented segmental motion. For other polymeric systems in which segmental motion is considerably constrained, this theory may be applied with some modifications. However, there is not enough necessary basic data available for general formulation yet.

\section{Conclusion}

(1) Two-component diffusivity for chain polymer system is derived theoretically as an extension of the free-volume theory of Bueche. With this equation, diffusivity, viscosity, temperature and molecular weight are correlated in a simple form.

(2) The behavior of diffusivity of non-polymeric and polymeric molecules in a polymeric medium is found to be classified into three regions by consideration of the entanglement effect.

(3) These theoretical equations are shown to be in good agreement with the experimental results in PDS systems, in which an anomalous behavior of diffusivity has been pointed out. The anomaly is explained in this work.

\section{Acknowledgement}

The authors wish to express their appreciation to Dr. Shoji Kimura for valuable discussion. They acknowledge with thanks the support of their work by the Asahi Glass Foundation for the Contribution to Industrial Technology.

\section{Nomenclature}

$$
\begin{aligned}
& \begin{array}{lll}
a & = & \text { length of a segmental unit }
\end{array} \\
& b \quad=\text { association parameter } \\
& D \quad=\text { diffusivity } \quad\left[\mathrm{cm}^{2} / \mathrm{sec}\right] \\
& D_{1,2}=\text { diffusivity of component } 1 \text { through medium } \\
& \text { component } 2 \\
& \begin{array}{lll}
f_{0} & =\text { molecular friction factor } \quad[\mathrm{g} / \mathrm{sec}]
\end{array} \\
& f_{o s}=\text { segmental friction factor } \quad[\mathrm{g} / \mathrm{sec}] \\
& k=\text { Boltzmann constant } \quad[\mathrm{erg} / \mathrm{deg}] \\
& M \quad=\text { molecular weight } \quad[\mathrm{g} / \mathrm{mol}] \\
& M_{s e g} \quad=\text { molecular weight corresponding to segmental unit } \\
& M e \quad=\text { molecular weight corresponding to several segmental } \\
& \text { units between entangling sites [g/mol] } \\
& N_{A}=\text { Avogadro number } \quad[1 / \mathrm{mol}] \\
& N_{A} \quad=\text { number of segmental units in a molecule }[-] \\
& N^{*} \quad=\text { modified number of segmental units in a molecule } \\
& r \quad=\text { radius of molecule } \quad[\mathrm{cm}] \\
& T=\text { temperature } \quad\left[{ }^{\circ} \mathrm{K}\right] \\
& t=\text { time [sec] } \\
& V \quad=\text { molar volume } \quad\left[\mathrm{cm}^{3} / \mathrm{mol}\right] \\
& V_{f} \quad=\text { molar free volume } \quad\left[\mathrm{cm}^{3} / \mathrm{mol}\right] \\
& V_{\text {seg }}=\text { molar volume per segmental unit } \quad\left[\mathrm{cm}^{3} / \mathrm{mol}\right] \\
& v_{f} \quad=\text { free volume per molecule or segmental unit }\left[\mathrm{cm}^{3}\right] \\
& v^{*} \quad=\text { critical free volume } \quad\left[\mathrm{cm}^{3}\right] \\
& <\text { Greek }> \\
& \begin{array}{ll}
\beta^{*} & =\text { defined by Eq. } \\
\delta & =\text { mean jump distance }
\end{array} \\
& \delta \quad=\text { mean jump distance } \\
& \eta \quad=\text { viscosity } \quad[\mathrm{g} / \mathrm{cm} \cdot \mathrm{sec}] \\
& \rho \quad=\text { density }\left[\mathrm{g} / \mathrm{cm}^{3}\right] \\
& \sigma \quad=\text { slippage factor } \\
& \phi \quad=\text { mean jump frequency } \quad[1 / \mathrm{sec}] \\
& \phi_{o} \quad=\text { proportionality constant } \quad[1 / \mathrm{sec}] \\
& <\text { Subscript }> \\
& p=\text { polymer } \\
& s \quad=\text { segment } \\
& 1,2=\text { component } 1 \text { and } 2 \text {; component } 1 \text { for penetrant } \\
& \text { molecule; component } 2 \text { for medium molecule }
\end{aligned}
$$

\section{Literature Cited}

1) Bueche, F.: "Physical Properties of Polymers", John Wiley \& Sons (1962)

2) Bueche, F.: J. Chem. Phys., 21, 1850 (1953); 24, 418 (1956); 
30, 748 (1959)

3) Bueche, F.: J. Chem. Phys., 20, 1959 (1952); 25, 599 (1956)

4) Castagnoli, G. G.: Physica, 30, 953 (1964)

5) Cohen, M. H. and D. Turnbull: J. Chem. Phys. 31, 1164 (1959)

6) Crank, J. and G. S. Park: "Diffusion in Polymers", Academic Press (1968)

7) Debye, P.: J. Chem. Phys., 14, 636 (1946)

8) Dullien, F. A. L.: A. I. Ch. E. J., 18, 62 (1972)

9) Einstein, A.: Ann. d. Phys., 17, 549 (1905); "Investigations on the Theory of the Brownian Movement", Dover (1956)

10) Eyring, H.: J. Chem. Phys., 4, 283 (1936)

11) Ferrell, R. T. and D. M. Himmelblau: A. I. Ch. E. J., 13, 702 (1967)

12) Gainer, J. L.: I. E. C. Fundamentals, 5, 436 (1966)

13) Glasstone, S., K. J. Laidler and H. Eyring: "The Theory of Rate Processes", McGraw-Hill (1941)

14) Hayduk, W. and S. C. Cheng: Chem. Eng. Sci., 26, 635 (1971)

15) Hill, N. E.: Proc. Phys. Soc. (London), 67B, 149 (1954), ibid., 68B, 209 (1955)

16) Hiranuma, M.: Kagaku Kögaku, 35, 997 (1971)

17) Houghton, G: J. Chem. Phys., 40, 1628 (1964)

18) Kamal, M. R. and L. Canjar: A.I. Ch. E. J., 8, 329 (1962)

19) King, C. J., L. H. Sueh and K. W. Mao: J. Chem. Eng. Data, 10, 348 (1965)

20) Li, J. C. M. and P. Chang: J. Chem. Phys., 23, 518 (1955)
21) Lusis, M. A. and G. A. Ratcliff: Can. J. Chem. Eng., 46, 385 (1968)

22) McLaughlin, E.: Trans. Faraday Soc., 55, 28 (1959)

23) Olander, D. O.: A. I. Ch.E. J., 9, 207 (1963)

24) Othmer, D. F. and M. S. Thakar: Ind. Eng. Chem., 45, 589 (1953)

25) Reddy, K. A. and L. K. Doraiswamy: I. E. C. Fundamentals, 6, $77(1967)$

26) Ree, F. H., T. Ree and H. Eyring: Ind. Eng. Chem., 50, 1036 (1958)

27) Rice, S., J. G. Kirkwood: J. Chem. Phys., 31, 584, 575, 901 (1959)

28) Rochow, E. G.: "Chemistry of the Silicons", John Wiley \& Sons (1951)

29) Scheibel, E. G.: Ind. Eng. Chem., 46, 2007 (1954)

30) Shin-etsu Chemical Industry Co.: Silicone KF96 Catalogue P6-3A:Technical Data Catalogue T6-4D: T6-7B; T6-8B

31) Sitaraman, R.: A.I. Ch. E.J., 8, 277 (1962)

32) Sitaraman, R., S. H. Ibrahim and N. R. Kuldoor: J. Chem. Eng. Data, 8, 198 (1963)

33) Tanner, J. E.: Macromolecules, 4, 748 (1971)

34) Tanner J. E.: private communication

35) Watanabe, H. and T. Miyauchi: to be submitted

36) Wilke, C. R.: Chem. Eng. Prog., 45, 218 (1949)

37) Wilke, C. R. and P. Chang: A.I. Ch.E.J., 1, 264 (1955)

\title{
APPLICATION OF THE VAN DER WAALS ONE FLUID THEORY TO PREDICT VAPOR-LIQUID EQUILIBRIA*
}

\author{
Eiji SARASHINA, YasuHiKo ARAI AND \\ Shozaburo SAITO \\ Department of Chemical Engineering, Tohoku University, \\ Sendai, Japan
}

\begin{abstract}
The van der Waals one-fluid theory was applied to mixtures of simple molecules and to systems containing carbon dioxide.

The excess properties of mixtures of simple molecules, $G^{E}, H^{E}$ and $V^{E}$, were well correlated by introducing correction factors, $\xi_{12}$ and $\eta_{12}$, for the Lorentz-Berthelot rules for the potential parameters between unlike molecules. Further, these parameters were useful in correlating the vapor-liquid equilibria for mixtures of simple molecules.

Among systems containing carbon dioxide, the vapor-liquid equilibria for carbon dioxide-light hydrocarbon systems and carbon dioxide-fluorocarbon systems were also well correlated by introducing the correction factor, $\xi_{12}$. On the other hand, systems consisting of carbon dioxide and simple molecules could be successfully correlated by adjusting the critical temperature of the light component.
\end{abstract}

\footnotetext{
* Received on August 24, 1972

A part of this paper has been published at the 4th Autumn Meeting of the Soc. of Chem. Engrs., Japan, at Hiroshima, Oct., 1970.

厂980 仙台市花巻字青葉 東北大学工学部化学工学科 斉藤正三郎
}

\section{Introduction}

The applicability of the corresponding-states principle to mixtures has been studied by several workers. In applying this principle to mixtures, they usually define the pseudopotential parameters for mixtures 